\title{
HOLOMORPHIC MAPPINGS OF BOUNDED DISTORTION
}

\section{A. MARDEN AND S. RICKMAN ${ }^{1}$}

ABSTRACT. Nonelementary holomorphic mappings $\mathrm{C}^{n} \rightarrow \mathrm{C}^{n}, n \geq 2$, cause severe geometric distortion near $\infty$ in sharp contrast to the case $n=1$ when there is no distortion.

The theory of quasiconformal and more generally quasiregular mappings $\mathbf{R}^{m} \rightarrow \mathbf{R}^{m}$ has been successful in recent years (see, for example, [4]) in drawing global topological consequences from the condition of uniformly bounded local distortion (the precise definition will be given below). With $\mathrm{H}$. Wu [9] it is natural to ask what this condition of bounded distortion implies for holomorphic mappings $\mathbf{C}^{n} \rightarrow \mathbf{C}^{n}$, regarding these as special cases of mappings $\mathbf{R}^{2 n} \rightarrow \mathbf{R}^{2 n}$. Wu [7], [8] considered such mappings in the course of his investigation of value distribution theory. The purpose of this note is to point out that the bounded distortion condition, imposed merely in a neighborhood of $\infty$, in the context of holomorphic mappings of $\mathrm{C}^{n}, n \geq 2$, is extremely restrictive: only affine mappings have this property.

Definition. A continuous map $f: D \rightarrow \mathbf{R}^{m}$ of a domain $D \subset \mathbf{R}^{m}$ is quasiregular if (a) $f$ is absolutely continuous on lines with $L_{\text {loc }}^{m}$ generalized partial derivatives, and (b) for some $1 \leq K<\infty$,

$$
\left|f^{\prime}(x)\right|^{m} \leq K J(x, f) \text {, a.e. }
$$

Here $f^{\prime}(x)$ denotes the Jacobian matrix, $\left|f^{\prime}(x)\right|$ its norm as a linear transformation, and $J(x, f)$ its determinant. A quasiregular map which is a homeomorphism is called quasiconformal.

Heuristically the definition says that a nonconstant quasiregular map sends tiny spheres to tiny ellipsoids for which the ratio of the longest to shortest axis is uniformly bounded by $K$.

Received by the editors August 6, 1973.

AMS (MOS) subject classifications (1970). Primary $32 \mathrm{H} 99$.

Key words and phrases. Quasiregular holomorphic mapping.

${ }^{1}$ Authors supported in part by the National Science Foundation. 
For $m \geq 3$ it is true [5] (see al so [3]). that a nonconstant quasiregular map $f: D \rightarrow \mathbf{R}^{m}$ for which $K=1$ is the restriction to $D$ of a Möbius transformation.

Definition. A holomorphic map $F: D \rightarrow \mathrm{C}^{n}$ of a domain $D \subset \mathrm{C}^{n}$ is called quasiregular if the induced map $f: D^{\prime} \rightarrow \mathbf{R}^{2 n}$ of the corresponding domain $D^{\prime} \subset \mathbf{R}^{2 n}$ is quasiregular, that is, if $f$ satisfies (1) with $m=2 n$.

We remark that this is the same as Wu's definition of 'quasiconformal holomorphic maps"' [7, p. 229].

Theorem. A nonconstant holomorphic map $F: \mathrm{C}^{n} \rightarrow \mathrm{C}^{n}, n \geq 2$, which is quasiregular in the complement of some polydisk, is of the form $F(x)=$ $A x+b$ where $A$ is a nonsingular $n \times n$ matrix and $b \in \mathbf{C}^{n}$.

Proof. The proof is based on two facts for quasiregular maps $f$ of a domain $D$ in $\mathbf{R}^{m}, m \geq 3$. (a) If it is sufficiently smooth, $f$ is a local homeomorphism [2, p. 95]. (b) If $\zeta$ is an isolated boundary point of $D$ and $f$ is a local homeomorphism in $D$ then $f$ can be extended to be a quasiregular local homeomorphism in $D \cup\{\zeta\}[1]$. Both the definition of quasiregularity and these two facts have natural analogs for the one point compactification of $\mathbf{R}^{m}$ and it is actually in this context that they will be applied.

In our situation then let $f: \mathbf{R}^{2 n} \rightarrow \mathbf{R}^{2 n}$ be the map induced by $F$. Since $f$ is quasiregular outside a sufficiently large ball we conclude from above that $f$ has a "removable singularity" at $\infty$. Consequently $[6,18.4]$ f $f$ satisfies a condition

$$
|f(x)| \leq C|x|^{a}, \quad|x|>R
$$

for some $a, C>0$ and $R<\infty(|\cdot|$ denotes the euclidean norm).

Retuming to $F=\left(F_{1}, \cdots, F_{n}\right),(2)$ implies that each component $F_{i}$ is a polynomial. The complex Jacobian determinant $I(x, F)$ is also a polynomial. Therefore there are only two possibilities: either (i) $\{x: I(x, F)=0\}$ is an algebraic variety of dimension $\geq 1$ and, in particular, is not compact, or (ii) $I(x, F)$ is a constant $\neq 0$.

Case (i) can be ruled out because $F$ is a local homeomorphism outside a sufficiently large polydisk and hence must have nonvanishing Jacobian there. Consequently case (ii) occurs. Then (1) implies that all the partial derivatives of $f$ and hence of $F$ are constants since they are polynomials (recall that $J(x, f)=|g(x, F)|^{2}$ ). Therefore $F$ has the desired form, completing the proof. 


\section{REFERENCES}

1. S. Agard and A. Marden, A removable singularity theorem for local homeomorphisms, Indiana Univ. Math. J. 20 (1970/71), 455-461. MR 43 \#2209.

2. P. T. Church, Differentiable open maps on manifolds, Trans. Amer. Math. Soc. 109 (1963), 87-100. MR 27 \#4245.

3. F. W. Gehring, Rings and quasiconformal mappings in space, Trans. Amer. Math. Soc. 103 (1962), 353-393. MR 25 \#3166.

4. O. Martio, S. Rickman and J. Väisälä, Topological and metric properties of quasiregular mappings, Ann. Acad. Sci. Fenn. Ser. A I No. 488 (1971). MR 45 \#8830.

5. Ju. G. Rèštnjak, On the stability of conformal mappings in multidimensional spaces, Sibirsk. Mat. Ž. 8 (1967), 91-114 = Siberian Math. J. 8 (1967), 69-85. MR $35 \# 367$.

6. J. Väisälä, Lectures on n-dimensional quasiconformal mappings, Lecture Notes in Math., no. 229, Springer-Verlag, Berlin and New York, 1971.

7. H. Wu, Normal families of holomorphic mappings, Acta Math. 119 (1967), 193-233. MR 37 \#468.

8. - Remarks on the first main theorem in equidistribution theory. III, J. Differential Geometry 3 (1969), 83-94. MR 43 \#2247c.

9. - Remarks on the first main theorem in equidistribution theory. IV, J. Differential Geometry 3 (1969), 433-446. MR 43 \#2247d.

SCHOOL OF MATHEMATICS, UNIVERSITY OF MINNESOTA, MINNEAPOLIS, MINNESOTA 55455

DEPARTMENT OF MATHEMATICS, UNIVERSITY OF HELSINKI, HELSINKI, FINLAND 